\title{
Expression of GATA3 and Cytokeratin 14 in Urinary Bladder Carcinoma (Histopathological and Immunohistochemical Study)
}

\author{
Nora Elzohery (iD*, Nourelhoda Sayed Ismael, Rasha Ahmed Khairy, Somia A. M. Soliman \\ Department of Pathology, Faculty of Medicine, Cairo University, Giza, Egypt
}

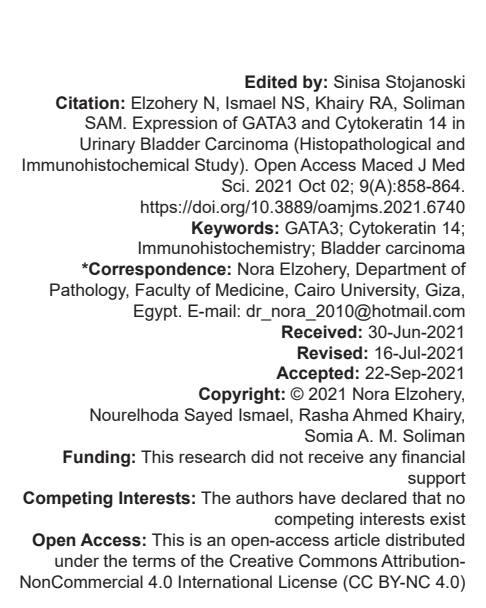

\section{Introduction}

Bladder cancer is common in the developed world and it is about $3 \%$ of global cancer diagnoses [1]. In Egypt, bladder cancer accounts for approximately $19 \%$ of the total incidence of cancers, and it is one of the common types of cancer in men and the second most common in females [2].

The urothelium of the bladder has a remarkable ability for divergent differentiation resulting in many histologic variants of urothelial cell origin. The variant histology carries worse prognosis than pure UC. These tumors present at a higher stage and are more likely to develop metastatic disease [3]. The outcomes varied with divergent histologies and appropriate treatment should be based on the histological finding [4].

The presence of squamous differentiation (SD) in bladder urothelial carcinoma (UC) was associated with high risk of recurrence, poor clinicopathological features, and worse clinical outcome for diseasefree survival. SD should be considered as a marker for treatment and prognosis for patients with bladder cancer [5].
GATA binding protein 3 is 1 of 6 members of zinc finger transcription factor family, was primarily recognized as a T-cell lineage-specific factor, and identified as an important transcription factor in hematopoiesis. It was then discovered that GATA3 has a significant role in the regulation of proliferation and differentiation in various nonhematopoietic tissues as mammary epithelial, bone, and parathyroid gland [6]. GATA3 mutations, loss of expression, or overall expression of GATA factors have all been associated with different types of cancers in humans. GATA3 is one of the most recently recognized urothelial-associated immunohistochemical markers which has been reported to be useful for assisting in the differential diagnosis of UCs [7]. It is also recognized as a marker of luminal subtype in bladder cancer [8].

Cytokeratin 14 (CK14) is a type I acidic keratin which is expressed in mitotically active basal cells of the stratified epithelium, as it promotes proliferation and differentiation and supports structural integrity. It was defined as the most primitive/least differentiated basaltype UC. CK 14 positive UC might have a tendency to be chemoresistant [9]. CK14 immunostaining is sufficiently sensitive to identify early squamous metaplasia which is not yet evident in the examined routine $H$ and $E$ stained sections [10]. 
The present work aimed to evaluate the immunohistochemical expression of GATA3 in UC and its sensitivity in distinguishing pure UC from UC with $\mathrm{SD}$, investigation of CK14 as a diagnostic marker for detection of squamous cell carcinoma (SCC) and SD of $\mathrm{UC}$ of the urinary bladder and correlation of GATA3 and CK14 expressions with available clinicopathological data.

\section{Material and Methods}

\section{Retrieval of cases}

The material of this retrospective study was collected as 60 archived cases of malignant epithelial neoplasm of urinary bladder at the Pathology Department, Faculty of Medicine, Cairo University, between January 2016 and November 2017. The cases were as follows: 42 cases obtained from radical cystectomies and 18 cases obtained from transurethral resection of tumor. The authors obtained the approval of the ethical committee in the faculty of Medicine, Cairo University.

Inclusion criteria included cases of urinary bladder carcinoma that underwent either radical cystectomy or cystoscopic biopsy. Exclusion criteria included cases of other malignant bladder neoplasm as sarcoma and lymphoma, cases of inadequate muscle proper, or cases with missed data.

\section{Histopathological examination}

- Each paraffin block was cut by rotatory microtome at $4 \mu \mathrm{m}$ thickness then mounted on glass slide and stained by hematoxlyin and eosin for histopathological examination and on charged slide for immunostaining

- $\quad$ Histological classification according to the World Health Organization recommendations [11]. Histological grading according to the World Health Organization recommendations [12].

- Extent of tumor invasion and nodal involvement were staged according to the American Joint Committee on Cancer's AJCC Cancer Staging [13].

- $\quad$ Associated schistosomal (Bilharzial) infestation based on detection of schistosoma ova in tissues.

\section{evaluation \\ Immunohistochemical staining and}

Immunohistochemical staining

using

BenchMark XT (Ventana) autostainer for
Rabbit monoclonal concentrated antibody against human Cytokeratin 14, clone EP61, Cat. \#RM0075, 1 ml. To confirm CK14 immunospecificity, a case of CK14 positive basal layer of prostatic tissue was used as a positive control which appears as brown cytoplasmic staining.

Mouse monoclonal concentrated antibody against GATA binding protein 3, clone L50-823, Cat. \#CM $405 \mathrm{~A}, 0.1 \mathrm{ml}$. To confirm GATA3 immuno-specificity, a case of GATA 3 positive breast cancer tissue was used as a positive control which appears as brown nuclear staining.

\section{GATA3 immunostaining interpretation}

Nuclear staining for GATA3 was graded as weak, moderate, or strong, and focal ( $\leq 20 \%$ of cells) or non-focal ( $>20 \%$ of cells) [14].

\section{CK14 immunostaining interpretation}

Positive CK14 immunostain appears as brown cytoplasmic staining. The assessment included the following: Total immunostaining score (TIS) was calculated by multiplying percentage score (PS), and intensity score (IS): PS: $0=$ no positive cells, $1=$ any positive cell up to $10 \%, 2=10-50 \%, 3=51-80 \%$, and $4=$ more than $80 \%$.

IS: $0=$ no color reaction, $1=$ mild intensity, $2=$ moderate intensity, and $3=$ strong intensity.

TIS: $0-1=$ negative, $2-3=$ mild, $4-8=$ moderate, and 9-12 = strong [15].

\section{Statistical analysis}

- Data management and analysis were performed using Statistical Package for Social Sciences (SPSS) vs. 21.

- $\quad$ Numerical data were summarized using means and standard deviations or medians and ranges. Categorical data were summarized as percentages. Comparisons between the 2 groups with respect to normally distributed numeric variables were done. For categorical variables, differences were analyzed with (Chi-square) test.

- $\quad$ All $p$-values are two-sided. $p<0.05$ were considered significant.

\section{Results}

Sixty studied cases ranging from 25 to 82 years old with mean age 58 years \pm 11 years. The majority 
of studied cases were males, with a percentage of $81.7 \%$, while females represented $18.3 \%$ of cases. Male: female ratio was $4.4: 1$. The tumor was present as single mass in most $(91.7 \%)$ of the cases. The studied cases were classified as Ta; T1; T2a; T2b; T3a; T3b and T4a were 15\%; 15\%; 3.3\%; 15\%; 5\%; 33.3\% and $13.3 \%$ respectively. T3b stage was the most common encountered in the studied cases (20 cases; $33.3 \%$ ).

The histological types were 13 SCC cases and 47 UC cases $(78.3 \%)$, classified into 17 papillary UC cases, 14 pure UC cases, 14 cases of UC with SD, and only two cases UC with glandular differentiation Figure 1.

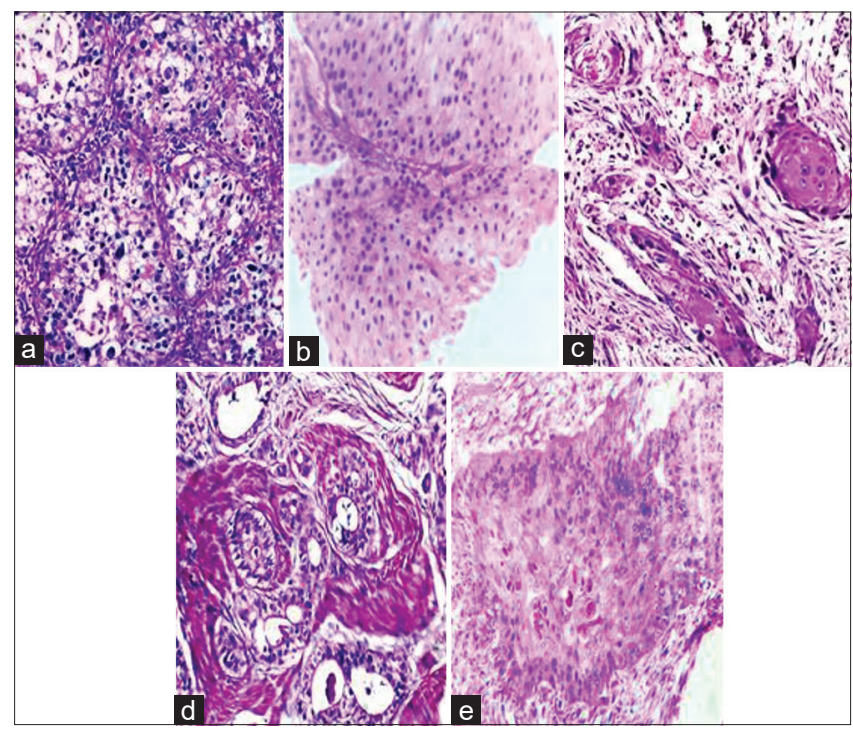

Figure 1: (a) Invasive pure urothelial carcinoma, hematoxylin and eosin ( $H$ and $E$ ) ( $\times 400$ original magnification. (b) Non invasive papillary urothelial carcinoma, $H$ and $E$ ( $\times 400$ original magnification. (c) Invasive urothelial carcinoma with squamous differentiation, $H$ and $E(\times 400$ original magnification). (d) Invasive urothelial carcinoma with glandular differentiation, $H$ and $E$ ( $\times 400$ original magnification). (e) Invasive squamous cell carcinoma, $H$ and $E$ ( $\times 400$ original magnification)

Thirty-three cases out of total of 47 cases of UC were high grade $(70.2 \%)$ and fourteen cases were low grade $(29.8 \%)$. All (13) cases of SCC were moderately differentiated Figure 2.

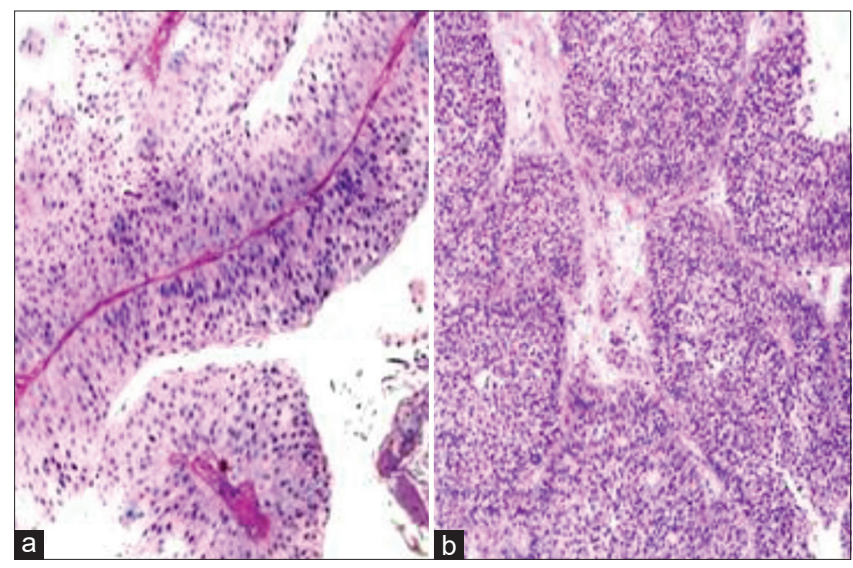

Figure 2: (a) Non invasive papillary urothelial carcinoma, low grade, hematoxylin and eosin ( $H$ and $E$ ) ( $\times 200$ original magnification). (b) Non nvasive papillary urothelial carcinoma, high grade, $H$ and $E$ $(\times 200$ original magnification)
Forty-two cases (70\%) showed negative GATA3 expression while 18 cases (30\%) showed positive GATA3 expression. Of the positive cases, $38.9 \%$ demonstrated mild positive staining, $38.9 \%$ demonstrated strong positive staining and $22.2 \%$ had moderate staining. $77.8 \%$ of the positive cases showed nonfocal staining. One hundred percent $(n=13)$ of SCC cases, $100 \%(n=14)$ of UC with SD cases and $85.7 \%(n=12)$ of pure UC cases showed negative GATA3 expression while $88.2 \%(n=15)$ of papillary UC cases and $14.3 \%(n=2)$ cases of pure UC showed positive GATA3 expression. The relation between the histological subtype and GATA3 expression intensity was statistically significant $(p<0.001)$ Figure 3 .

There was statistically significant correlation between tumor stage and GATA3 expression, 55.6\% of stage Ta cases showed strong positive GATA3 expression and $100 \%$ of stage $4 \mathrm{a}$ cases showed negative expression ( $p<0.001)$. A significant inverse

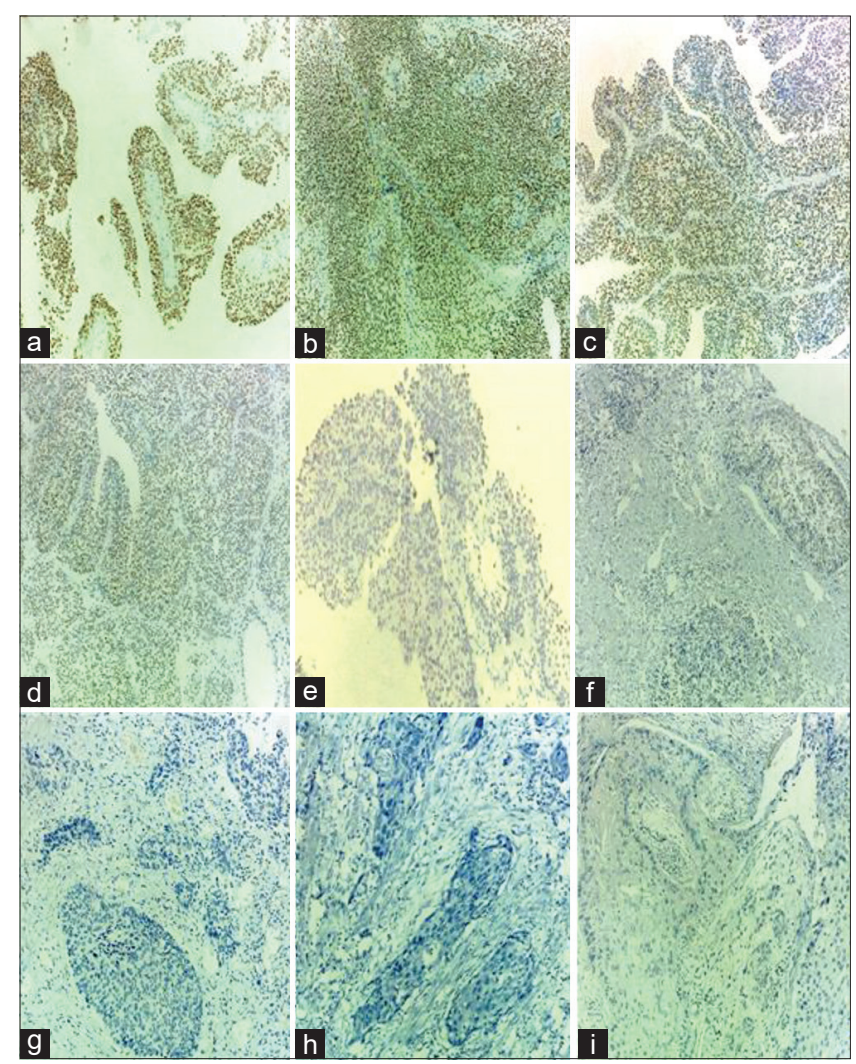

Figure 3: (a) Non-Invasive papillary urothelial carcinoma showing strong nonfocal GATA3 expression ( $\times 100$ original magnification). (b) Invasive papillary urothelial carcinoma showing strong nonfocal GATA3 expression ( $\times 100$ original magnification). (c) Invasive papillary urothelial carcinoma showing moderate nonfocal GATA3 expression ( $\times 100$ original magnification). (d) Invasive papillary urothelial carcinoma showing weak nonfocal GATA3 expression $(\times 100$ original magnification). (e) Invasive papillary urothelial carcinoma showing weak focal GATA3 expression $(\times 400$ original magnification). (f) Invasive pure urothelial carcinoma showing weak focal GATA3 expression ( $\times 400$ original magnification). (g) Invasive pure urothelial carcinoma showing negative GATA3 expression ( $\times 400$ original magnification). (h) Invasive urothelial carcinoma with squamous differentiation showing negative GATA3 expression ( $\times 400$ original magnification). (i) Invasive squamous cell carcinoma showing negative GATA3 expression ( $\times 400$ original magnification) 
correlation between tumor histological grade and GATA3 expression intensity was detected where $50 \%$ of low-grade cases showed strong positive GATA3 expression and $84.8 \%$ of high-grade cases showed negative expression $(p<0.001)$.

In the current study, 22 cases $(36.7 \%)$ showed positive CK14 expression, while 38 cases (63.3\%) showed negative CK14 expression. Among the positive group, 15 cases (25\%) showed strong positive expression, 4 cases $(6.7 \%)$ showed moderate positive expression and three cases $(5 \%)$ showed mild positive expression. One hundred percent of SCC cases showed strong positive CK14 expression as well as (100\%) of all types of UC other than UC with SD showed negative expression while $64.3 \%$ cases of UC with SD showed positive CK14 expression. A statistically significant correlation between the histological subtype and CK14 expression was detected $(p<0.001)$ Figure 4.

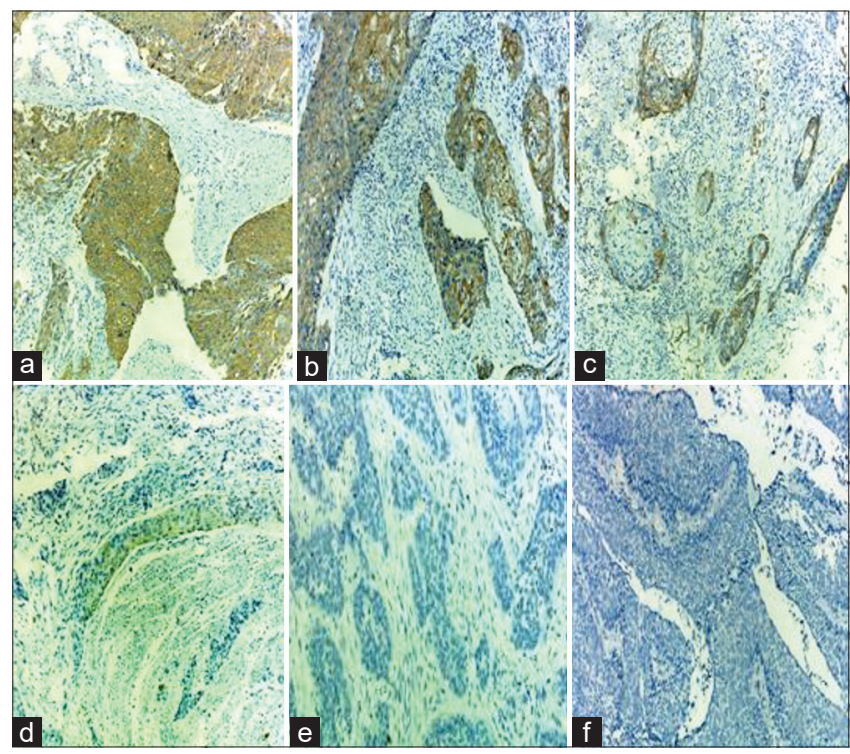

Figure 4: (a) Invasive squamous cell carcinoma showing strong positive Cytokeratin 14 (CK14) expression (×100 original magnification). (b) urothelial carcinoma with squamous differentiation showing strong positive CK14 expression ( $\times 200$ original magnification). (c) Invasive urothelial carcinoma with squamous differentiation showing moderate positive CK14 expression ( $\times 100$ original magnification). (d) Invasive urothelial carcinoma with squamous differentiation showing weak positive CK14 expression ( $\times 100$ original magnification). (e) Invasive urothelial carcinoma with squamous differentiation showing negative CK14 expression ( $\times 200$ original magnification). (f) Non-invasive papillary urothelial carcinoma showing negative CK14 expression ( $\times 100$ original magnification)

A statistically significant correlation between tumor stage and CK14 expression was detected. Seventy-five percentage of stage T4a cases showed strong positive CK14 expression ( $p<0.001)$. There was significant correlation between CK14 expression and the tumor size as $50 \%$ of tumors with a size $>3.6 \mathrm{~cm}$ showed strong positivity for CK14, while only $5.9 \%$ of tumors $\leq 3.6 \mathrm{~cm}$ showed strong positivity $(p=0.003)$.

A statistically significant inverse correlation between CK14 expression and GATA3 expression was detected. All GATA3 positive cases showed negative CK14 expression and near half of CK14 positive cases showed negative GATA3 expression $(p<0.001)$ Figure 5.

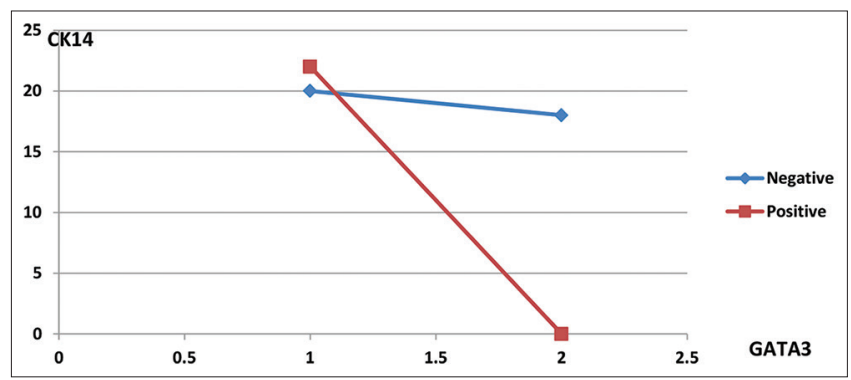

Figure 5: Correlation between GATA3 expression and Cytokeratin 14 expression in the studied cases

Details of the relation of GATA3 expression with clinicopathological variables of studied cases are summarized in Table 1.

Table 1: Relation of GATA3 intensity expression with clinicopathological variables

\begin{tabular}{|c|c|c|c|c|c|}
\hline & \multicolumn{2}{|c|}{ Negative } & \multicolumn{2}{|c|}{ Positive } & \multirow[t]{2}{*}{$p$ value } \\
\hline & Count & $\%$ & Count & $\%$ & \\
\hline \multicolumn{6}{|l|}{ Size categories } \\
\hline$\leq 3.6 \mathrm{~cm}$ & 17 & 50.0 & 17 & 50.0 & \multirow[t]{2}{*}{$<0.001^{*}$} \\
\hline$>3.6 \mathrm{~cm}$ & 25 & 96.2 & 1 & 3.8 & \\
\hline \multicolumn{6}{|l|}{ Histological type } \\
\hline Papillary urothelial carcinoma & 2 & 11.8 & 15 & 88.2 & \multirow[t]{5}{*}{$<0.001^{*}$} \\
\hline Pure urothelial carcinoma & 12 & 85.7 & 2 & 14.3 & \\
\hline $\begin{array}{l}\text { Urothelial carcinoma with squamous } \\
\text { differentiation }\end{array}$ & 14 & 100.0 & 0 & 0.0 & \\
\hline $\begin{array}{l}\text { Urothelial carcinoma with glandular } \\
\text { differentiation }\end{array}$ & 1 & 50.0 & 1 & 50.0 & \\
\hline Squamous cell carcinoma & 13 & 100.0 & 0 & 0.0 & \\
\hline \multicolumn{6}{|l|}{ Grade } \\
\hline Low & 1 & 7.1 & 13 & 92.9 & \multirow[t]{3}{*}{$<0.001^{*}$} \\
\hline High & 28 & 84.8 & 5 & 15.2 & \\
\hline Moderate & 13 & 100.0 & 0 & 0.0 & \\
\hline \multicolumn{6}{|l|}{ Invasion } \\
\hline Invasive & 41 & 80.4 & 10 & 19.6 & \multirow[t]{2}{*}{$<0.001^{*}$} \\
\hline Non-invasive & 1 & 11.1 & 8 & 88.9 & \\
\hline \multicolumn{6}{|l|}{ Tumour Stage (T) } \\
\hline Ta & 1 & 11.1 & 8 & 88.9 & \multirow[t]{7}{*}{$<0.001^{*}$} \\
\hline T1 & 2 & 22.2 & 7 & 77.8 & \\
\hline T2a & 2 & 100.0 & 0 & 0.0 & \\
\hline $\mathrm{T} 2 \mathrm{~b}$ & 8 & 88.9 & 1 & 11.1 & \\
\hline ТЗа & 3 & 100.0 & 0 & 0.0 & \\
\hline T3b & 18 & 90.0 & 2 & 10.0 & \\
\hline T4a & 8 & 100.0 & 0 & 0.0 & \\
\hline
\end{tabular}

Details of the relation of CK14 expression with clinicopathological variables of studied cases are summarized in Table 2.

Details of the relation of histologic subtype with tumor invasion and stage of studied cases are summarized in Table 3.

Details of the correlation between GATA3 expression and CK14 expression in the studied cases are summarized in Table 4.

\section{Discussion}

In our study, we analyzed the association between percentage of SD and tumor stage and found it presented usually at advanced stage, which is in agreement with meta-analysis done by Lin et al. (2019) 
who showed patients with UC with SD were associated with the unfavorable clinicopathological characteristics of tumor, such as high percentage of pT3/T4 and high tumor grade [5].

In the present work, 42 cases $(70 \%)$ showed negative GATA3 expression, while 18 cases $(30 \%)$ showed GATA3 expression. Of the UC cases, 38.3\% demonstrated positive staining.

The incidence in this study is lower than obtained by Agarwal et al. (2019), Mohammad et al. (2016), and Wang et al. (2019) in which positive GATA3 expression is (77\%), (70.8\%), and $(87.9 \%)$, respectively [7], [16], [17].

The discrepancies observed in these results may be related to patient selection bias and lack of standard immunohistochemical assays, including different properties of the antibodies.

The results in the present study revealed that 13 cases $(100 \%)$ of SCC specimens and 14 cases $(100 \%)$ of UC with SD specimens showed negative GATA3 staining. While 18 cases $(54.5 \%)$ of UC specimens other than UC with SD showed positive expression and most of the positive cases (83.3\%) were papillary UC.

Table 2: Relation of CK14 expression with clinicopathological variables

\begin{tabular}{|c|c|c|c|c|c|}
\hline & \multicolumn{4}{|l|}{ CK14 } & \multirow[t]{3}{*}{$p$ value } \\
\hline & \multicolumn{2}{|c|}{ Negative } & \multicolumn{2}{|c|}{ Positive } & \\
\hline & Count & $\%$ & Count & $\%$ & \\
\hline \multicolumn{6}{|l|}{ Size categories } \\
\hline$\leq 3.6 \mathrm{~cm}$ & 27 & 79.4 & 7 & 20.6 & \multirow[t]{2}{*}{$0.003^{*}$} \\
\hline$>3.6 \mathrm{~cm}$ & 11 & 42.3 & 15 & 57.7 & \\
\hline \multicolumn{6}{|l|}{ Histological type } \\
\hline Papillary urothelial carcinoma & 17 & 100.0 & 0 & 0.0 & \multirow[t]{5}{*}{$<0.001^{*}$} \\
\hline Pure urothelial carcinoma & 14 & 100.0 & 0 & 0.0 & \\
\hline $\begin{array}{l}\text { Urothelial carcinoma with squamous } \\
\text { differentiation }\end{array}$ & 5 & 35.7 & 9 & 64.3 & \\
\hline $\begin{array}{l}\text { Urothelial carcinoma with glandular } \\
\text { differentiation }\end{array}$ & 2 & 100.0 & 0 & 0.0 & \\
\hline Squamous cell carcinoma & 0 & 0.0 & 13 & 100.0 & \\
\hline \multicolumn{6}{|l|}{ Grade } \\
\hline Low & 14 & 100.0 & 0 & 0.0 & \multirow[t]{3}{*}{$<0.001^{*}$} \\
\hline High & 24 & 72.7 & 9 & 27.3 & \\
\hline Moderate & 0 & 0.0 & 13 & 100.0 & \\
\hline \multicolumn{6}{|l|}{ Invasion } \\
\hline Invasive & 29 & 56.9 & 22 & 43.1 & \multirow{2}{*}{$0.020^{*}$} \\
\hline Non-invasive & 9 & 100.0 & 0 & 0.0 & \\
\hline \multicolumn{6}{|l|}{ Tumour Stage (T) } \\
\hline $\mathrm{Ta}$ & 9 & 100.0 & 0 & 0.0 & \multirow[t]{7}{*}{$<0.001^{*}$} \\
\hline T1 & 9 & 100.0 & 0 & 0.0 & \\
\hline T2a & 2 & 100.0 & 0 & 0.0 & \\
\hline $\mathrm{T} 2 \mathrm{~b}$ & 5 & 55.6 & 4 & 44.4 & \\
\hline Т3а & 2 & 66.7 & 1 & 33.3 & \\
\hline T3b & 11 & 55.0 & 9 & 45.0 & \\
\hline T4a & 0 & 0.0 & 8 & 100.0 & \\
\hline
\end{tabular}

Nearly similarly GATA3 expression was reported by Liang et al. (2014) showed that 13 cases (93\%) of SCC showed negative GATA3 and (70\%) of pure UC cases showed positive expression. On the other hand, Helmy et al. (2015) found that (95\%) of SCC cases showed negative GATA3 expression, while 70\% of UC with SD and (100\%) of pure UC cases showed positive expression [18], [19].

A significant correlation between GATA3 immunohistochemical expression and histological tumor subtypes of bladder carcinoma ( $p<0.001)$ was found in this work, i.e. the GATA3 is useful marker for urothelial origin especially in papillary UC and can be used in differentiating between UC and SCC. This is in concordance to results reported by Liang et al. (2014), who found that GATA3 expression in SCC was significantly lower than that of pure UC. This was also in agreement with study done by Helmy et al. (2015) [18], [19].

In this study, there was an inverse relationship between GATA3 immunohistochemical expression and UC grade $(p<0.001)$ i.e. the GATA3 expression decreased or lost with high tumor grade. It was seen that $(71.4 \%)$ of lowgrade tumors showed moderate to strong immunoreactivity for GATA3 expression as compared to (84.8\%) of highgrade tumors which didn't show any reactivity.

This inverse association is consistent with that reported by Agarwal et al. (2019) who found a statistically significant correlation $(p<0.001)$ between histological grade and GATA3 expression pattern, where $100 \%$ of low-grade tumors showed moderateto-strong GATA3 expression and $70 \%$ of high-grade tumors showed weak expression. Rest of the highgrade tumors showed negative expression [7]

This was also in agreement with studies done by Miyamoto et al. (2012), Kamel et al. (2019), and Li et al. (2014) who reported that GATA3 was down-regulated in high-grade invasive bladder tumors [20], [21], [22].

There was a statistically significant association between GATA3 expression and the tumor stage in the current study $(p<0.001)$ where high stage tumor (pT4a) showed loss of GATA3 expression. The same finding reported by Miyamoto et al. (2012) [20].

Results of the studies done by Abdullah et al. (2018) and Liang et al. (2014) were different as they

Table 3: Relation of histologic subtype with tumor invasion and stage

\begin{tabular}{|c|c|c|c|c|c|c|c|}
\hline & \multicolumn{2}{|c|}{$\begin{array}{l}\text { Urothelial carcinoma with } \\
\text { squamous differentiation }\end{array}$} & \multicolumn{2}{|c|}{ Squamous cell carcinoma } & \multicolumn{2}{|c|}{$\begin{array}{l}\text { Urothelial carcinoma other than urothelial carcinoma } \\
\text { with squamous differentiation }\end{array}$} & \multirow[t]{2}{*}{$P$ value } \\
\hline & Count & $\%$ & Count & $\%$ & Count & $\%$ & \\
\hline \multicolumn{8}{|l|}{ Invasion } \\
\hline Invasive & 14 & 27.5 & 13 & 25.5 & 24 & 47.1 & $0.012^{*}$ \\
\hline Non-invasive & 0 & 0.0 & 0 & 0.0 & 9 & 100.0 & \\
\hline \multicolumn{8}{|l|}{ Tumour Stage (T) } \\
\hline $\mathrm{Ta}$ & 0 & 0.0 & 0 & 0.0 & 9 & 100.0 & $<0.001^{*}$ \\
\hline T1 & 0 & 0.0 & 0 & 0.0 & 9 & 100.0 & \\
\hline T2a & 0 & 0.0 & 0 & 0.0 & 2 & 100.0 & \\
\hline $\mathrm{T} 2 \mathrm{~b}$ & 2 & 22.2 & 2 & 22.2 & 5 & 55.6 & \\
\hline Т3a & 2 & 66.7 & 1 & 33.3 & 0 & 0.0 & \\
\hline T3b & 7 & 35.0 & 5 & 25.0 & 8 & 40.0 & \\
\hline T4a & 3 & 37.5 & 5 & 62.5 & 0 & 0.0 & \\
\hline
\end{tabular}


reported that GATA-3 expression was not significantly associated with tumor stage. This incompatibility may be explained by the difference in sample size of the subgroups [18], [23].

Table 4: Correlation between GATA3 expression and CK14 expression in the studied cases

\begin{tabular}{lllllll}
\hline & \multicolumn{2}{l}{ CK14 } & & & p value \\
\cline { 2 - 3 } & Negative & & & \\
\cline { 2 - 3 } & Count & $\%$ & & Count & $\%$ & \\
\hline GATA3 & 20 & 47.6 & & 22 & 52.4 & $<0.001^{*}$ \\
Negative & 20 & 100.0 & & 0 & 0.0 & \\
Positive & 18 & & & &
\end{tabular}

In the present study, the tumor size had significant correlation with GATA3 expression ( $P$ value $<0.001)$ i.e. the larger tumor size showed loss of GATA3 expression. These findings were different from that reported by Mohammad et al. (2016) who found high GATA3 expression was associated with larger tumor size in invasive UC. While Agarwal et al. (2019) had seen no association between tumor size and GATA3 expression [7], [16].

The present study showed a statistically significant correlation between the histological subtype and CK14 expression ( $p<0.001$ ) detected as CK14 showed positive cytoplasmic staining in 9/14 (64.3\%) cases of UC with SD and $13 / 13(100 \%)$ cases of pure SCC and negative in 33/33(100\%) cases of UC other than UC with SD. CK14 had sensitivity (100\%) in SCC, sensitivity (64.3\%), and specificity (100\%) in UC with SD. CK14 staining appeared only in areas of SD and not in areas of UC. It showed strong expression in $2 / 14$ cases $(14.3 \%)$, moderate expression in $4 / 14$ cases (28.6\%), and mild expression in $3 / 14$ cases $(21.4 \%)$.

This is in agreement with El-Nashar et al. (2016) who showed CK14 expression in 100\% of cases with SCC, but showed CK14 expression in (7/7) cases of invasive UC with SD, in addition to $4 / 24(16.7 \%)$ of cases of pure UC, and Moselhy et al. (2018), who reported positive expression in $23 / 31(74.2 \%)$ cases of UC with SD, 5/26 (19.2\%) cases of pure UC, and in all cases (16/16) of SCC. Also, Helmy et al. (2015) reported CK14 expression in $100 \%$ of cases with SCC and $100 \%$ of cases with invasive UC with SD, and negative in $100 \%$ of cases of pure UC [10], [15], [19].

Gulmann et al. (2013) showed CK14 expression in $100 \%$ of cases with SCC, $74 \%$ of cases of invasive UC with SD, and $27 \%$ of cases of pure UC. Hammam et al. (2014) reported that CK14 was positive in all the pure SCCs and also was positive in $58 \%$ of UCs [24], [25].

The discrepancies observed in these results in the positivity of some cases of pure UC may be related to probable foci of early squamous phenotypic switch. Other different findings can be explained by the fact that they used a larger number of cases.

In the current study, CK14 expression showed significant statistical association with tumor stage $(p=0.001)$ and this was in agreement with Jangir et al. (2019) who reported muscle-invasive bladder cancer expressing only basal markers (CK14 and CK5/6) presented at an advanced stage with frequent SD and showed a trend towards shorter overall survival [26].

\section{Conclusion}

The results of this study conclude that GATA3 is a potential immunohistochemical marker for urothelial origin, especially for papillary UC. The intensity of expression decreased and lost with high-grade tumors. and thus it constitutes a potentially promising novel prognostic marker in patients with UC.

This study confirms that CK14 is a highly specific and sensitive immunohistochemical marker indicative of SCC and SD in UC suggesting the possible role of CK14 as diagnostic marker for them. It can be used to differentiate UC with SD from pure UC.

Combination of GATA3 and CK14 could be very helpful in detecting pure primary SCC, pure UC, and UC with SD.

Wider scale studies using larger sample sizes and long-term follow-up are further needed to establish the prognostic significance of GATA3 expression. Further studies are needed to detect the value of CK14 and GATA3 immunohistochemistry expression in the molecular classification of bladder carcinoma.

\section{References}

1. Saginala K, BarsoukA, Aluru JS, Rawla P, Padala SA, BarsoukA Epidemiology of bladder cancer. Med Sci. 2020;18(1):15. https:// doi.org/10.3390/medsci8010015

2. Elsharkawi F, Elsabah M, Shabayek M, Khaled H. Urine and serum exosomes as novel biomarkers in detection of bladder cancer. Asian Pac J Cancer Prev. 2019;20(7):2219-24. https:// dx.doi.org/10.31557\%2FAPJCP.2019.20.7.2219 PMid:31350988

3. Matulay JT, Narayan VM, Kamat A. Clinical and genomic considerations for variant histology in bladder cancer. Curr Oncol Rep. 2019;21:23. https://doi.org/10.1007/s11912-019-0772-8 PMid:30806832

4. Veskimäe E, Espinos EL, Bruins HM, Yuan Y, Sylvester R, Kamat AM, et al. What is the prognostic and clinical importance of urothelial and nonurothelial histological variants of bladder cancer in predicting oncological outcomes in patients with muscle-invasive and metastatic bladder cancer? A European association of urology muscle invasive and metastatic bladder cancer guidelines panel systematic review. 2019;2(6):625-42. https://doi.org/10.1016/j.euo.2019.09.003

PMid:31601522

5. Lin X, Deng T, Wu S, Sharron X, Lin S, Wang D, et al. The 
clinicopathological characteristics and prognostic value of squamous differentiation in patients with bladder urothelial carcinoma: A metaanalysis. World J Urol. 2019;38(2):323-33. https://doi.org/10.1007/s00345-019-02771-1

PMid:31011874

6. Wang C, Yang S, Jin L, Dai G, Yao Q, Xiang H, et al. Biological and clinical significance of GATA3 detected from TCGA database and FFPE sample in bladder cancer patients. Onco Targets Ther. 2020;13:945-58. https://doi.org/10.2147/OTT.S237099 PMid:32099398

7. Agarwal H, Babu S, Rana C, Kumar, Singhai, Shankhwar SN, et al. Diagnostic utility of GATA3 immunohistochemical expression in urothelial carcinoma. Indian $\mathrm{J}$ Pathol Microbiol. 2019;62(2):244-50.

PMid:30971548

8. Robertson G, Kim J, Al-Ahmadie H, Bellmun J, Guo G, Cherniack $A D$, et al. Comprehensive molecular characterization of muscle-invasive bladder cancer. Cell. 2017;171(3):540-56. https://doi.org/10.1016/j.cell.2017.09.007

PMid:30971548

9. Jung M, Jang I, Kim K, Moon KC. CK14 expression identifies a basal/squamous-like type of papillary non-muscle-invasive upper tract urothelial carcinoma. Front Oncol. 2020;10:623. http://doi:10.3389/fonc.2020.00623

PMid:32391279

10. El-Nashar A, Sotohy TM, Mohammad EM, Mahmoud SF, Badawy A. The use of desmocollin-2 and cytokeratin-14 for detection of squamous cell carcinoma and squamous differentiation in urothelial carcinoma. Med $\mathrm{J}$ Cairo Univ. 2016;849(1):387-94.

11. Moch H, Humphrey PA, Ulbright TM, Reuter VE. Tumors of the urinary tract. In: WHO Classification of Tumours of the Urinary System and Male Genital Organs. $4^{\text {th }}$ ed. Lyon: IARC; 2016. p. 78. https://doi.org/10.1016/j.eururo.2016.02.029

12. Humphrey PA, Moch $\mathrm{H}$, Cubilla AL, Ulbright TM, Reuter VE. The 2016 WHO classification of tumours of the urinary system and male genital organs-part B: Prostate and bladder tumours. Eur Urol. 2016;70(1):106-19. https://doi.org/10.1016/j. eururo.2016.02.028 PMid:26996659

13. American Cancer Society. Last Medical Review. Atlanta, Georgia: American Cancer Society; 2019.

14. Chang A, Amin A, Gabrielson E, Illei P, Roden RB, Sharma R, et al. Utility of GATA3 immunohistochemistry in differentiating urothelial carcinoma from prostate adenocarcinoma and squamous cell carcinoma of the uterine cervix, anus, and lung. Am J Surg Pathol. 2012;36(10):1472-6. https://doi.org/10.1097/ pas.0b013e318260cde7 PMid:22982890

15. Moselhy AA, Aiad HA, El Rebey HS, Mahmoud SE. Immunohistochemical expression of cytokeratin 14 and association with the extent of squamous differentiation in urothelial carcinoma. Menoufia Med J. 2018;31:826-33.

16. Mohammed $\mathrm{KH}$, Siddiqui MT, Cohen C. GATA3 immunohistochemical expression in invasive urothelial carcinoma. Urol Oncol. 2016;34(10):432.e9-13. https://doi. org/10.1016/j.urolonc.2016.04.016

PMid:27241168

17. Wang CC, Tsai YC, Jeng YM. Biological significance of GATA3, cytokeratin 20 , cytokeratin $5 / 6$ and p53 expression in muscle invasive bladder cancer. PLoS One. 2019;14(8):e0221785. https://doi.org/10.1371/journal.pone.0221785 PMid:31469885

18. Liang $\mathrm{Y}$, Heitzjman J, Kamat AM, Dinney CP, Czerniak B, Guo CC. Differential expression of GATA-3 in urothelial carcinoma variants. Hum Pathol. 2014;45(7):1466-72. https:// doi.org/10.1016/j.humpath.2014.02.023 PMid:24745616

19. Helmy NA, Khalil HK, Kamel NN, AboelFadl DM. Role of GATA3, CK7, CK20 and CK14 in distinguishing urinary bladder squamous cell carcinoma and urothelial carcinoma with squamous differentiation. Egypt J Pathol. 2015;35(2):133-8. https://doi.org/10.1097/01.XEJ.0000472878.82311.73

20. Miyamoto H, Izumi K, Yao JL, Li Y, Yang Q, McMahon LA, et al. GATA binding protein 3 is down-regulated in bladder cancer yet strong expression is an independent predictor of poor prognosis in invasive tumor. Hum Pathol. 2012;43(11):2033-40. https://doi. org/10.1016/j.humpath.2012.02.011 PMid:22607700

21. Kamel NA, Abdelzaher E, Elgebaly O, Ibrahim SA. Reduced expression of GATA3 predicts progression in non-muscle invasive urothelial carcinoma of the urinary bladder. J Histotechnol. 2020;43(1):21-8. https://doi.org/10.1080/01478 885.2019.1667126

PMid:31551051

22. Li Y, Ishiguro $\mathrm{H}$, Kawahara T, Miyamoto $\mathrm{Y}$, Izumi $\mathrm{K}$, Miyamoto $\mathrm{H}$. GATA3 in the urinary bladder: Suppression of neoplastic transformation and down-regulation by androgens. Am J Cancer Res. 2014;4(5):461-73.

PMid:25232488

23. Abdullah W, Kerbel $H$, Husseini R. Applying gata3 in differentiating urothelial carcinoma from prostatic adenocarcinoma: An immunohistochemical study. Asian J Pharm Clin Res. 2018;11(12):292-5. https://doi.org/10.22159/ ajpcr.2018.v11i12.28232

24. Gulmann C, Paner GP, Parakh RS, Hansel DE, Shen SS, Ro JY, et al. Immunohistochemical profile to distinguish urothelial from squamous differentiation in carcinomas of urothelial tract. Hum Pathol. 2013;44(2):164-72. https://doi.org/10.1016/j. humpath.2012.05.018 PMid:22995333

25. Hammam O, Wishahi M, Khalil H, Ganzouri H, Badawy M, Elkhquly A, et al. Expression of cytokeratin 7, 20, 14 in urothelial carcinoma and squamous cell carcinoma of the Egyptian urinary bladder cancer. J Egypt Soc Parasitol. 2014;44(3):733-40. PMid:25643514

26. Jangir H, Nambirajan A, Ranjit AS, Sahoo K, Dinda AK, Nayak $B$, et al. Prognostic stratification of muscle invasive urothelial carcinomas using limited immunohistochemical panel of Gata3 and cytokeratins 5/6, 14 and 20. Ann Diagn Pathol. 2019;43:151397. https://doi.org/10.1016/j.anndiagpath.2019.08.001

PMid:31494492 